\title{
Efficient reduction of PLI in ECG signal using new variable step size least mean fourth adaptive algorithm
}

\author{
T. Gowri ${ }^{1}$, Rajesh Kumar P. ${ }^{2}$, D.V.R. Koti Reddy ${ }^{3}$ \\ ${ }^{1}$ Department of Electronics and Communication Engineering, GIT, GITAM University, India \\ ${ }^{2}$ Department of Electronics and Communication Engineering, AUCE, Andhra University, India \\ ${ }^{3}$ Department of Instrument Technology, AUCE, Andhra University, India
}

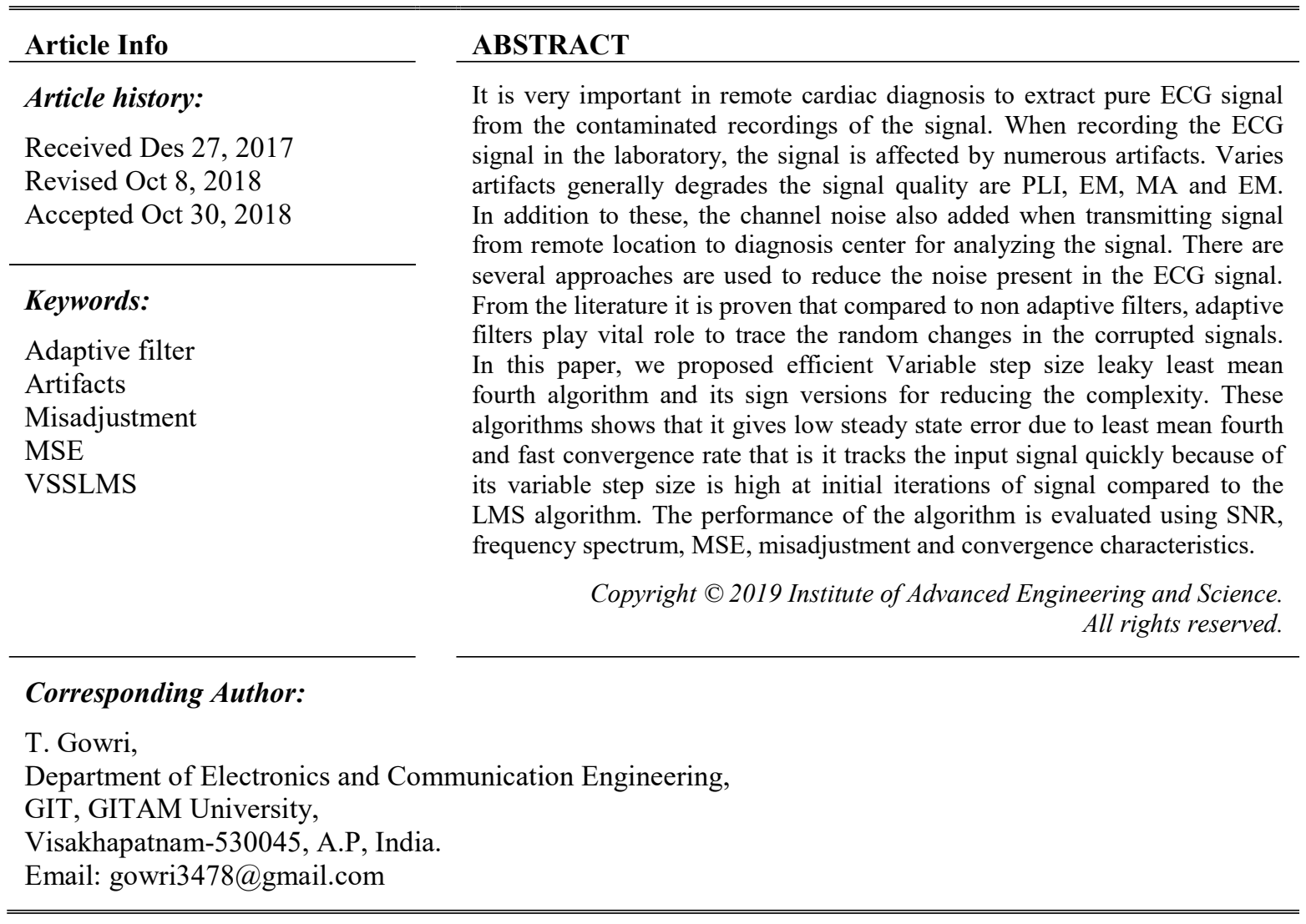

\section{INTRODUCTION}

ECG is one of the most direct ways to study the cardiac condition. Any cardiac abnormalities will be clearly seen through investigation of the ECG signals. This provides doctors with the most updated cardiac condition of the patient so that proper treatment can be given [1]. Biotelemetry is a technology where wireless communication is utilized in health care applications to transmit biological or physiological data to a distant place then ECG specialist has the capability to interpret the data and affect decision-making [2]. Telecardiology is a special branch of biotelemetry in which the ECG signals are processed wirelessly between the patient and diagnostic centre. While transmitting the ECG signal from patient end to diagnostic centre, several artifacts may add to ECG signal. These artifacts strongly affect/degrade the signal quality of ECG signals and masks tiny features [3, 4].

To improve the signal quality in terms of signal to noise ratio and to reduce noises present in the signal then different filtering techniques are used. In the literature there are several filtering techniques are used to remove the artifacts present in ECG signal [5-7]. Butt et al. [7], presented the PLI noise reduction in the ECG signal using SSRLS filter, in this, result analysis it gives the superior performance as compared with the notch filter of varying attenuation levels. Hamilton [8] compared the performance of adaptive and nonadaptive notch filters used to remove the single-frequency component present in PLI. Kadam et al. [9] described a PLI subtraction technique. In referred paper, different digital FIR windowing techniques are used 
for the cancellation of $60 \mathrm{~Hz}$ interference in ECG frequency content. The extracted samples give the better elimination of PLI noise.

Shin et al. [10] proposed a variable step size affine projection algorithm and a variable step size normalized LMS algorithm methods. In this simulation results analysis they proved that these algorithms to get faster convergence rate and less misadjustment value. Similarly, Vega et al. [11] presented a robust VSSNLMS algorithm for system identification and removal of echo's based on norm of the filter update. But complexity of this algorithm is large. Another VSSNLMS was developed for cancellation of residual echo's produced by the part of the systems by Paleologu et al. [12].

In this paper, we proposed a new Variable Step Size Least Mean Fourth (VSS-LMF) algorithm which gives less computational complexity and low misadjustment value. For further reducing computational complexity we applied signum function to this algorithm and derived three other algorithms. These four algorithms gives better signal to noise ratio compared to LMS algorithm and VSSLMS algorithm in terms of removal of artifacts present in the ECG signal. From the simulation results it is verified that VSS-Sign Regressor LMF algorithm gets better reduction of noise present in ECG signal compared to other derived algorithms and LMS algorithm.

\section{ADAPTIVE ALGORITHM}

The adaptive LMS algorithm was invented by Widrow and Hoff in 1959 [13, 14]. The LMS algorithm can be considered as the simplest form of steepest decent algorithm. The LMS algorithm is composed by two basic processes, Filtering process and an adaptive process. The structure of adaptive filter is shown in the Figure 1. From the Figure $x(j)$ the input signal, $d(j)$ is the desired signal, $y(j)$ is filter output and the error signal $e(j)$ is given to the input for tap weight vector control mechanism. The tap inputs $[x(j), x(j-1), \ldots, x(j-L+1)]$ are the elements of the L-by-1 tap input vector $x(j)$. Where $\mathrm{L}$ is the number of delay elements. Correspondingly, the tap weights $\boldsymbol{w}(j)=[w(j), w(j-1), \ldots, w(j-L+1)]^{T}$ form the elements of the L-by-1 tap-weight vector. The updated weight vector for the LMS adaptive algorithm in simplified form as (1).

$$
\boldsymbol{w}(j+1)=\boldsymbol{w}(j)+\mu \boldsymbol{x}(j) e(j)
$$

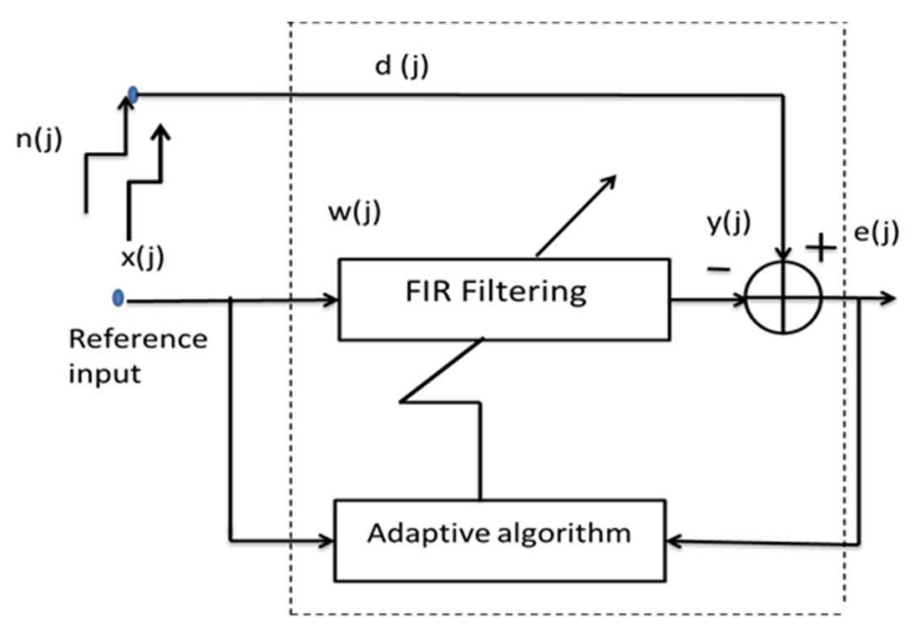

Figure 1. Adaptive filter structure

The parameter $\mu$ describes the rate at which convergence of the algorithm starts and finally reaches to Wiener optimum solution. The problem with LMS algorithm [13] is that selecting the step size parameter $\mu$ smaller then convergence will be slow but the steady state error will be small and vice versa. So selecting the proper value of $\mu$ is difficult in the LMS algorithm. Therefore the variable step-size gives a trade-off between steady state misadjustment and convergence rate of the algorithm. This problem motivates to use the step size as variable, rather than fixed $[15,16]$. Adaptive filters based on higher order (like fourth order) 
statistics gives better performance (less error) than the mean square like LMF [17] performs better than the mean square utilized in the LMS algorithm in particular situations. The small modification in (1), leads weight update equation for VSS-LMF algorithm which is defined as (2):

$$
\boldsymbol{w}(j+1)=\boldsymbol{w}(j)+\mu(j) \boldsymbol{x}(j) e^{3}(j)
$$

Where $\mu(j)$ is a scalar variable step-size parameter and is considered as (3):

$$
\mu(j)=\frac{1-\alpha}{1.5\left(1-\alpha^{j+1}\right)}
$$

where ' $j$ ' is the occurrence number in the iteration. (3) leads the fast convergence rate at the beginning of the signal occurrences due to a large variable step size, and decreasing the step size in the latter cases to a less misadjustment error ratio, and then finally it reaches to a steady state value. ' $\alpha$ ' is constant parameter, in this analysis the values chosen is 0.9 which gives better result. This decreasing step size is the main variation in the VSS-LMF which is not possible in LMS. The VSS-LMF algorithm (2) requires L+3 multiplication. These "multiply and accumulate" (MAC) operations are reduced by adding signum function to the (2). These reduced complexity algorithms are very useful to design digital signal processing devices and field programmable gate arrays. The complexity of the algorithm further reduced by using the signum function and it is defined as (4):

$$
\operatorname{sign}\{p(j)\}=\left\{\begin{aligned}
1: & p(j)>0 \\
0: & p(j)=0 \\
-1: & p(j)<0
\end{aligned}\right\}
$$

The signum (polarity) function can be applied to the data in the weight update (2), then new derived algorithm is VSS-Sign Regressor LMF algorithm and is given by (5):

$$
\boldsymbol{w}(j+1)=\boldsymbol{w}(j)+\mu(j) \operatorname{sign}(\boldsymbol{x}(j)) e^{3}(j)
$$

The number of multiplications required for VSS-SRLMF algorithm is 3, so compared to VSS-LMF algorithm ' $L$ ' number of multiplications reduced. So the complexity of the circuit is reduced for designing process. By applying signum function to the error function in the VSS-LMF algorithm, the new algorithm is named as VSS-Sign LMF algorithm and its weight update equation is as (6):

$$
\boldsymbol{w}(j+1)=\boldsymbol{w}(j)+\mu(j) \boldsymbol{x}(j) \operatorname{sign}\left(e^{3}(j)\right)
$$

The number of multiplications required for VSS-SLMF algorithm is $\mathrm{L}+1$. The signum can also be applied for both error and data functions in (2), the new algorithm is known as VSS-Sign Sign LMF algorithm is given by (7):

$$
\boldsymbol{w}(j+1)=\boldsymbol{w}(j)+\mu(j) \operatorname{sign}(\boldsymbol{x}(j)) \operatorname{sign}\left(e^{3}(j)\right) .
$$

The VSS-SSLMF algorithm requires only one multiplication, compared to all above algorithms this algorithm requires less complexity. So finally in this paper totally four algorithms developed, whose performance characteristics are analyzed by reducing artifact in the ECG signal.

\section{SIMULATION RESULT AND ANALYSIS}

The ability of varies derived algorithms are tested by taking real ECG signal from the MIT-BIH arthymia database [18] by adding different artifacts. In this data base there are totally 48 ECG records are digitized with a sampling frequency of 360 Hertz per channel. From the 48 ECG data records, 22 data's are from women and 25 data's are from men with different age groups are collected. From the ECG data records 201 and 202 data records are collected from the single person. The ECG signal is corrupted with a power line 
noise of amplitude $1 \mathrm{mV}$, with $60 \mathrm{~Hz}$ frequency applied as input signal. From the data base 4000 samples are collected for records 101, 102, 103, 104, 105, 108 and 207. The length of the filter chosen is five and step size chosen is 0.5 . The simulation results are obtained using MATLAB software. The frequency spectrums for different adaptive algorithms for cancellation of $60 \mathrm{~Hz}$ 's PLI noise are shown in Figure 2. From this figure, we can observe that VSS-LMF as shown in Figure $2 \mathrm{~d}$ algorithm gets high reduction of PLI noise compared to LMS algorithm as shown in Figure 2-c.

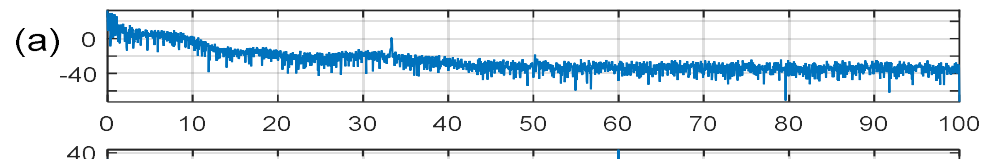

(b)
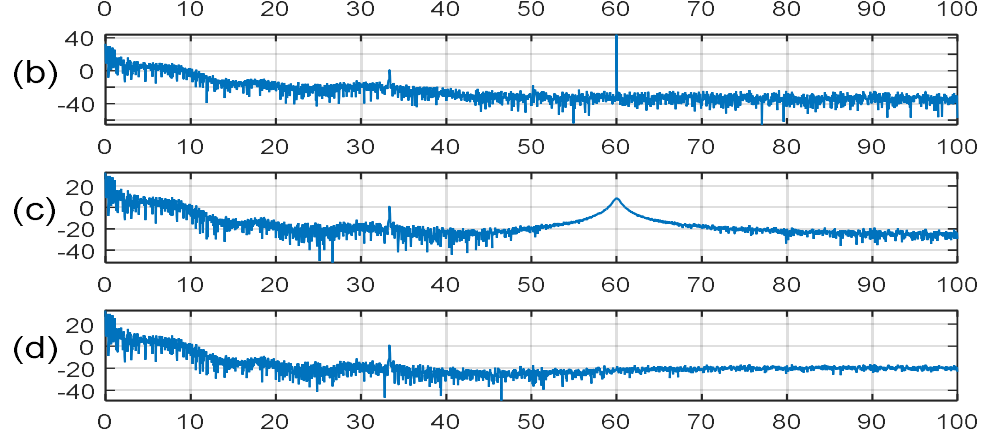

Figure 2. Typical frequency spectrums for PLI cancellation (a) Pure ECG signal, (b) Pure ECG signal with PLI, (c) Recovered signal using LMS algorithm, (d) Recovered signal using VSS-LMF-algorithm.

From the Figure 3(a) it shows that pure ECG signal is corrupted with PLI and Figure 3(b-e) shows that removal of PLI noise using different derived adaptive algorithms. From the figure we can see that at starting iterations there is small residue noise present, when number of iterations increases then noise elimination is more that is minimum steady state error we can get due to variable step size. It is observed that VSS-LMF and VSS-SRLMF algorithms reduce PLI noise as high compared to other two algorithms.

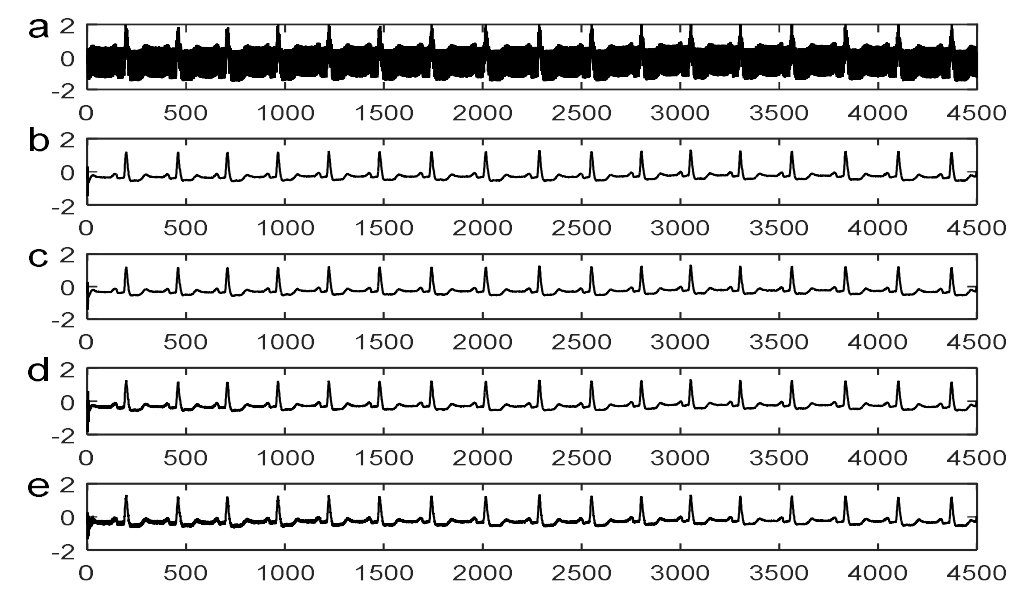

Figure 3. (a) ECG signal with PLI, (b) Filtered signal using VSS-LMF algorithm, (c) Filtered signal using VSS-SRLMF algorithm, (d) Filtered signal using VSS-SLMF algorithm, (e) Filtered signal using VSS-SSLMF algorithm

The performance of the removal of PLI using various algorithms is measured in terms of SNR, as shown in Table 1. From the Table 1, it is observed that all algorithms achieves maximum average SNR values which are higher value than the LMS [19]. Comparing all derived algorithms VSS-LMF algorithm gets maximum average SNR value of $12.7308 \mathrm{dBs}$, where as next VSS-SRLMF algorithm gets $12.6018 \mathrm{dBs}$, 
VSS-SLMF algorithm gets $8.0303 \mathrm{dBs}$ and VSS-SSLMF algorithm gets $8.9599 \mathrm{dBs}$. To enjoy the less computational complexity and also high SNR value nearer to VSS-LMF, then choose VSS-SRLMF algorithm is the best. For further reduction of complexity and greater than the LMS, SNR value then choose VSS-SSLMF algorithm.

Table 1. SNR contrast of VSS-LMF Based Adaptive Algorithms for PLI Removal

\begin{tabular}{ccccc}
\hline Rec. No & VSS-LMF & VSS-SRLMF & VSS-SLMF & VSS-SSLMF \\
\hline 101 & 12.5857 & 12.7968 & 6.681 & 8.0701 \\
102 & 12.6912 & 12.0536 & 8.5396 & 6.5072 \\
103 & 11.5402 & 11.6811 & 7.9506 & 8.6456 \\
104 & 13.4422 & 13.6118 & 9.0846 & 11.9711 \\
105 & 12.4371 & 11.2984 & 7.8346 & 7.7669 \\
108 & 13.1334 & 13.3758 & 7.2169 & 8.2264 \\
207 & 13.2864 & 13.3951 & 8.9052 & 11.5321 \\
Avg SNR & 12.7308 & 12.6018 & 8.0303 & 8.9599 \\
\hline
\end{tabular}

The convergence curves for different adaptive algorithms in terms number of iterations verses mean square error as shown in Figure 4. These characteristics are drawn for ECG record number 103. From the convergence characteristics it is observed that VSS-SRLMF algorithm gives fast convergence rate as compared to other adaptive algorithms and VSS-SLMF algorithm gets slow convergence rate.

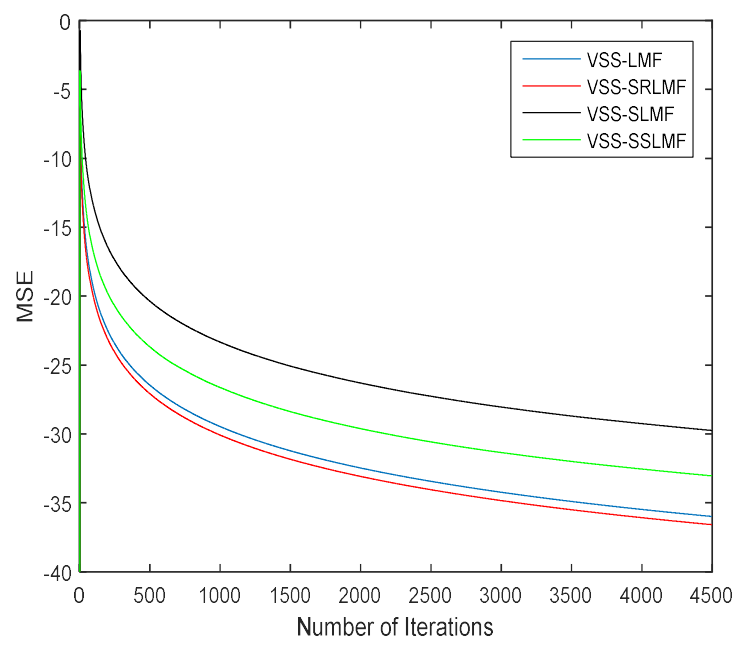

Figure 4. Figure convergence characteristics of VSS-LMF based adaptive algorithms

The Mean Square Error (MSE) for different adaptive algorithms for different records as shown in Table 2. From the Table 2, VSS-LMF algorithm gets 2.87e-04 MSE, which is low compared to other algorithms, then after VSS-SRLMF algorithm gets 3.43e-04 MSE which is also low compared to LMS and other sign based algorithms. The ratio of excess MSE to minimum MSE gives Misadjustment.

Table 2. MSE Comparison using Different Adaptive Algorithms

\begin{tabular}{ccccccccc}
\hline Algorithm & Rec No 101 & Rec No 102 & Rec No 103 & Rec No 104 & Rec No 105 & Rec No 108 & Rec No 207 & Avg MSE \\
\hline LMS & 0.0045 & 0.0045 & 0.0046 & 0.0045 & 0.0046 & 0.0045 & 0.0045 & 0.0045 \\
VSS-LMF & $2.4490 \mathrm{e}-04$ & $2.6034 \mathrm{e}-04$ & $3.4738 \mathrm{e}-04$ & $2.5216 \mathrm{e}-04$ & $3.9555 \mathrm{e}-04$ & $2.4229 \mathrm{e}-04$ & $2.6648 \mathrm{e}-04$ & $2.87 \mathrm{e}-04$ \\
VSS-SRLMF & $2.0842 \mathrm{e}-04$ & $2.3580 \mathrm{e}-04$ & $5.3246 \mathrm{e}-04$ & $2.3284 \mathrm{e}-04$ & $7.2581 \mathrm{e}-04$ & $2.1340 \mathrm{e}-04$ & $2.5309 \mathrm{e}-04$ & $3.43 \mathrm{e}-04$ \\
VSS-SLMF & 0.0058 & 0.0019 & 0.0021 & 0.0019 & 0.0041 & 0.0042 & 0.0021 & 0.0031 \\
VSS-SSLMF & 0.0030 & 0.0014 & $9.2203 \mathrm{e}-04$ & $5.0007 \mathrm{e}-04$ & 0.0041 & 0.0025 & $6.0824 \mathrm{e}-04$ & 0.0018 \\
\hline
\end{tabular}

The misadjustment values for different algorithms for the removal of PLI noise are shown in Table 3. From the table VSS-LMF and VSS-SRLMF algorithms gets very low misadjustment value as compared to the LMS, VSS-SLMF and VSS-SSLMS adaptive algorithms. 
Table 3. Misadjustment Comparison Using Different Adaptive Algorithms

\begin{tabular}{lcccccccc}
\hline Algorithm & Rec No 101 & Rec No 102 & Rec No 103 & Rec No 104 & Rec No 105 & Rec No 108 & Rec No 207 & $\begin{array}{c}\text { Avg } \\
\text { Misadjustment }\end{array}$ \\
\hline LMS & $1.3636 \mathrm{e}-04$ & $1.3903 \mathrm{e}-04$ & $1.4924 \mathrm{e}-04$ & $1.4951 \mathrm{e}-04$ & $1.4826 \mathrm{e}-04$ & $1.3674 \mathrm{e}-04$ & $1.4895 \mathrm{e}-04$ \\
VSS-LMF & $2.1881 \mathrm{e}-06$ & $3.7019 \mathrm{e}-06$ & $3.6629 \mathrm{e}-06$ & $3.9616 \mathrm{e}-06$ & $3.5829 \mathrm{e}-06$ & $2.5816 \mathrm{e}-06$ & $3.8307 \mathrm{e}-06$ & $3.36 \mathrm{e}-06$ \\
VSS- & $1.8081 \mathrm{e}-05$ & $1.6089 \mathrm{e}-05$ & $2.5076 \mathrm{e}-05$ & $2.0137 \mathrm{e}-05$ & $1.3680 \mathrm{e}-05$ & $1.6317 \mathrm{e}-05$ & $2.4819 \mathrm{e}-05$ \\
SRLMF & 0.0296 & $6.7637 \mathrm{e}-04$ & $3.8213 \mathrm{e}-04$ & $3.6240 \mathrm{e}-04$ & 0.0071 & 0.0077 & $3.8862 \mathrm{e}-04$ & $0.92-05$ \\
VSS-SLMF & 0.0185 & 0.0078 & $4.6409 \mathrm{e}-04$ & $2.0418 \mathrm{e}-04$ & 0.0196 & 0.0135 & $3.6326 \mathrm{e}-04$ & 0.0086 \\
VSS- & & & & & & & &
\end{tabular}

\section{CONCLUSION}

In this paper, weight update variants of New Variable Step Size-LMF based adaptive algorithms are developed. The variable of step size for each iteration leads to fast convergence. To achieve fast data transmission rate it is desirable to reduce the computational complexity. This can be done by signed algorithms which are derived from conventional algorithm. The three versions of signed algorithms are derived. So totally four adaptive noise cancellers are developed and tested on ECG signals for cancellation of PLI noise. Among these sign regressor VSS-LMF algorithms performs better SNR with less computational complexity as well as fast convergence as that of VSS-LMF algorithm. These derived algorithms can also be applied for other artifacts which will give better elimination of noise.

\section{REFERENCES}

[1] World Health Organization Report, "Prevention and Control of Non-Communicable Diseases: Implementation of Global Strategy," World Health Organization, Geneva, Available: https://apps.who.int/gb/ebwha/pdf_files/EB120/b120_22-en.pdf, 2007.

[2] D.C.Jeutter, "Telecommunications for Health Care," Biomedical Engg. Department, http://www.eng.mu.edu/biotelem/objectives.html.

[3] I. Guler and S. Kara, "A Low-Cost Biotelemetry System for Long Time Monitoring of Physiological Data," Journal of Medical Systems, vol. 20, no. 3, pp.151-156, 1996.

[4] H. Kim, R. F. Yazicioglu, T. Torfs, P. Merken, C. Van Hoof and H.J. Yoo, "An Integrated Circuit for Wireless Ambulatory Arrhythmia Monitoring Systems," in Proc. IEEE Int. Conf. of the EMBS Minneapolis, pp. 5409-5412, Sept. 2-6,2009.

[5] S. M. M. Martens, M. Mischi, S. Guid Oei and Jan W. M. Bergmans, "An Improved Adaptive Power Line Interference Canceller for Electrocardiography," IEEE Transactions on Biomedical Engineering, vol. 53, no. 11, pp. 2220-2231, 1996.

[6] T. Gowri, P. Rajesh Kumar "Improved RLS based Adaptive Filtering Technique to Enhance the Quality of ECG Signal”, in European Journal of Scientific Research, vo. 136, pp. 56-61, 2015.

[7] M. Butt, N. Razzaq, I. adiq, M. Salman and T. Zaidi, "PLI Removal From ECG Signal Using SSRLS Algorithm," in Proc. IEEE 9th Int. Colloquium on Processing and its Applications, pp. 95-98, Mar. 8-10, 2013.

[8] P.S. Hamilton, "A Comparison of Adaptive and Non adaptive Filters for Reduction of Power Line Interference in the ECG," IEEE Transactions on Biomedical Engineering, vol. 43, no. 1, pp. 105-109, 1996.

[9] Geeta Kadam, P.C.Bhaskar, "Reduction of PLI in ECG Signal Using FIR Filter," international Journal of Computational Engineering Research, vol. 2, no.2, pp.314-319, 2012.

[10] H. C. Shin, A. H. Sayed and Woo-Jin Song, "Variable Step-Size NLMS and Affine Projection Algorithms," IEEE Signal Processing Letters, vol. 11, no. 2, pp. 132- 135, 2004.

[11] L. R. Vega, Hernn Rey, J. Benesty and S. Tressens, "A New Robust Variable Step-Size NLMS Algorithm," IEEE Transaction on Signal Processing, vol. 56, no. 5, pp. 1878-1893, 2008.

[12] C. Paleologu, S. Ciochina and J. Benesty, "Variable Step-Size NLMS for Under-Modeling Acoustic Echo Cancellation," IEEE Signal Processing Letters, vol. 15, pp. 5-8, 2008.

[13] S. S. Haykin, “Adaptive Filter Theory,” Eaglewood Cliffs, N. J.: Prentice-Hall, 1986.

[14] B. Widrow and S.D. Stearns, "Adaptive Signal Processing," Eaglewood Cliffs, Prentice Hall, NJ 1985.

[15] T. Aboulnasr and K Mayyas, "A Robust Variable Step-Size LMS-Type Algorithm: Analysis and Simulations," IEEE Transactions on Signal Process, vol. 45, no. 3, pp. 631-639, 1997.

[16] X. Wu, L. Gao and Z. Tan, "An Improved Variable Step Size LMS Algorithm," in Proc. IEEE 2nd Int. Conf. on Measurement, Information and Control, vol. 01, pp. 533-536, Aug. 16-18, 2013.

[17] E. Walach and B. Widrow, "The Least Mean Fourth Adaptive Algorithm and Its Family," IEEE Transactions on Information Theory, vol. 30, pp. 275-283, 1984.

[18] http://www.physionet.org/physiobank/database/mitdb/-(Online).MIT-BIH Arrhythmia Database.

[19] Md. Zia. Ur. Rahman, K.M. Krishna, G.V.S Karthik, M Jhon Joseph and M Ajay Kumar, "Non Stationary Noise Cancellation in Speech Signals Using an Efficient Variable Step Size Higher Order Filter", International journal of Research and Reviews in computer sciences, vol.2, No.2, pp.414-422, 2011. 


\section{BIOGRAPHIES OF AUTHORS}
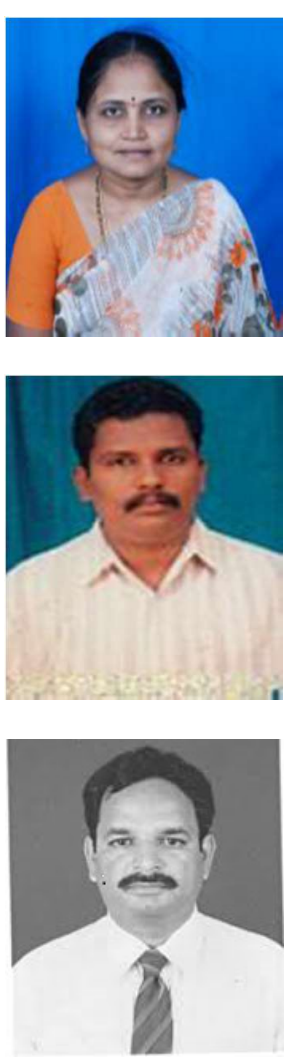

T. Gowri, She received B.Tech from Nagarjuna University, M. Tech from Jawaharlal Nehru Technological University anatapur and PhD from Jawaharlal Nehru Technological University kakinada. She is currently working as an Assistant Professor in the Department of Electronics and Communication Engineering, GIT, GITAM University, Visakhapatnam, A.P, India. Her research interests include Digital Information Systems and Computer Electronics, Digital Signal Processing and Information Security. She has more than 15 years experience of Teaching under graduate and post graduate level.

P. Rajesh Kumar, He received his Ph.D from Andhra University, Visakhapatnam, India. He is currently working as a Professor in the Department of Electronics and communication engineering depart. College of Engineering, Andhra University, Visakhapatnam, India. His field of interest includes Radar signal processing, Image Processing, Communications and antenna theory Applications. He has more than 20 years of teaching and 14 years of research experience. He published 35 research papers in international journals. He guided $10 \mathrm{Ph} . \mathrm{D}$ theses. He held different positions in his career like Head of the Department and Assistant Principal.

D.V.Rama Koti Reddy, He received his Ph.D from Andhra University, Visakhapatnam, India. He is currently working as a Professor and Head of department in the Department of Instrument Technology, College of Engineering, Andhra University, Visakhapatnam, India. He has more than 20 years of teaching and 10 years of research experience. He published 35 research papers in international journals. His field of interest includes Sensors and sensor networking, MEMS and Nano Technology and Signal Processing. 